\title{
Research on AMT Vehicle Control System Based on Neural Network
}

\author{
Yanning Wang ${ }^{\mathrm{a}}$ \\ Guangdong University of Science \& Technology, Dongguan, Guangdong, 523083, China. \\ a358986013@qq.com
}

Keywords: AMT vehicle control system; neural network; motor control.

\begin{abstract}
People often use AMT to summarize various changes and new systems brought about by traditional manufacturing technologies such as microelectronics, automation, and information technology. Specifically, it refers to the collective name of technologies, equipment, and systems that are created by integrating mechanical engineering technology, electronic technology, automation technology, and information technology. In this paper, the main research is based on neural network AMT car control system research. During the operation of the AMT vehicle system, when the driver manually shifts the gear, the coordination of the components of the power transmission system of the vehicle body is poor, and the driver frequently shifts the gear, resulting in a low safety factor. The static continuous shifting test is performed on the shifting mechanism. The test data shows that the addition of a neural network speeds up the motor's response to control signals.
\end{abstract}

\section{Introduction}

AMT uses advanced electronic technology to transform the traditional manual transmission, not only retaining the advantages of high efficiency and low cost of the original gear transmission, but also possesses all the advantages brought about by the automatic shifting of the hydraulic automatic transmission. It has been welcomed by all drivers with its unique economy, convenience, safety and comfort, and has become a hot spot for various countries to develop. Its disadvantage is non-power shifting, which can be compensated by electronic control software. The research in this paper is based on the influence of neural network on AMT car control system, which makes AMT car control system more in line with people's needs [1].

\section{Amt System Works}

The basic control principle of the AMT transmission system is: the ECU is integrated according to the driver's manipulation (operation of the throttle pedal, brake pedal, steering wheel, gear selector) and the running state of the vehicle (vehicle speed, engine speed, transmission input shaft speed) Judgment, determination of the driver's intentions and road conditions, the use of appropriate control laws, issued control instructions, with the help of the corresponding executive agencies, the vehicle's powertrain joint operation[2]. AMT maintains the basic structure of the original mechanical transmission, the more commonly used imported AT (hydraulic torque converter automatic transmission), has the advantages of high transmission efficiency, compact structure, low cost, easy to manufacture, reliable work and convenient operation.

\section{Neural Network Application in Amt Control System}

The control system of a car is a complex process that is influenced and controlled in many ways, and it is also affected by many external environments. Modeling of such nonlinear systems has always been a major difficulty in the research of automotive systems. The emergence of neural networks provides a new idea for AMT control systems.

\subsection{BP Network Structure}

BP in the BP neural network is a shorthand for Back Propagation. It was first proposed by Rumelhart, McCelland, and other scientists in 1986. Rumelhart also published a very famous article "Learning representations by back-propagating errors" in Nature. . 
With the migration of the times, the BP neural network theory has been continuously improved and updated. Now it has undoubtedly become one of the most widely used neural network models. We know that the transmission of human brain information and the response to external stimuli are controlled by neurons. The human brain consists of billions of such neurons. These neurons are not isolated and closely related. Each neuron is connected to an average of several thousand neurons and thus constitutes a neural network of the human brain. Stimuli propagate in the neural network following certain rules. A neuron does not react every time it receives stimulation from other nerves. It will first accumulate the stimuli coming from its neighboring neurons and, at a certain time, will generate its own stimuli and pass it on to some of its adjacent neurons. The billions of neurons that work in this way constitute the reaction of the human brain to the outside world. The mechanism by which the human brain learns external stimuli is by regulating the connections between these neurons and their strength [3]. Of course, the above is actually a simplified biological model of the true neural work of the human brain. Using this simplified biological model, it can be extended to machine learning and described as an artificial neural network. The BP neural network is one of them. Figure 1 below shows the structural analysis of neurons.

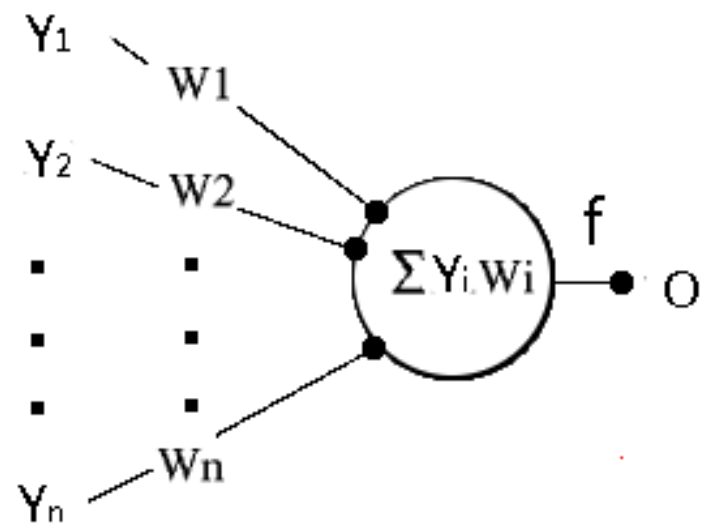

Figure 1. Neurons

When individual neurons accumulate, they form a network. The composition of the BP neural network is shown in Figure 2 below.

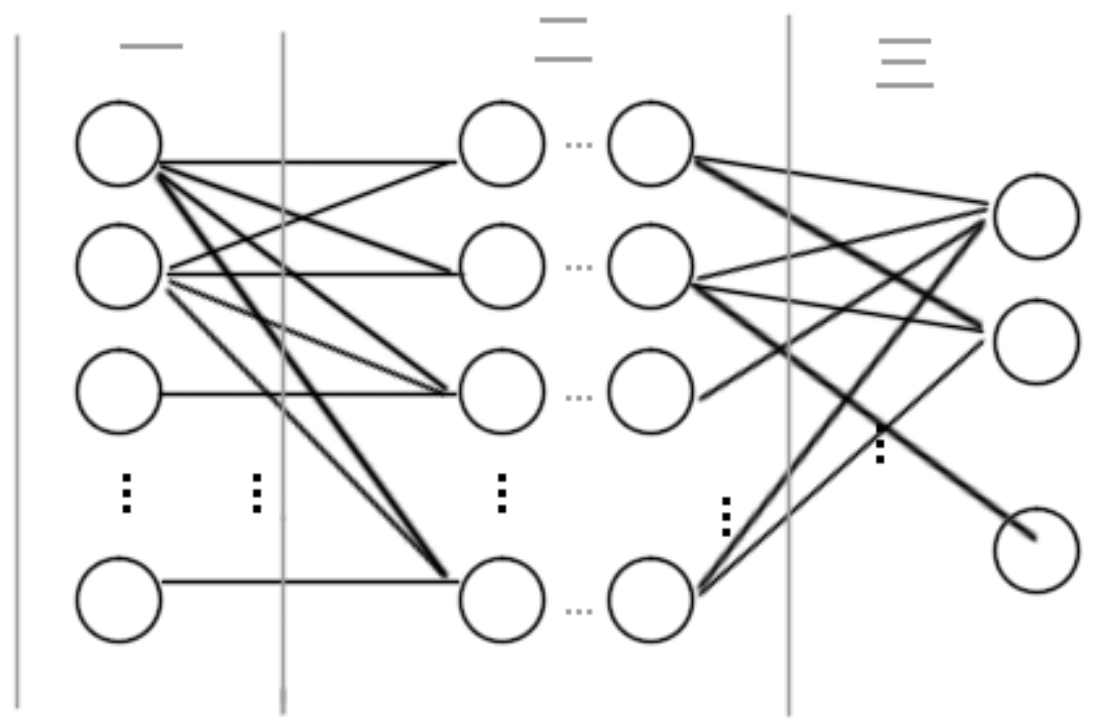

Figure 2. BP neural network

In terms of the first region, they correspond to external stimuli, are sources of stimuli, and deliver stimuli to neurons. Therefore, the first region is designated as the input layer. The second area indicates that the neurons transfer stimuli to each other in the human brain. Therefore, the second area is named hidden layer. The third area represents the response of the neurons to the outside world after multiple levels of mutual transmission, so the third area is named as the output layer. 
The simple description is that the input layer passes the stimulus to the hidden layer. The hidden layer passes the stimulus to the output layer through the strength (weight) of the connection between the neurons and the transfer rule (activation function). The output layer arranges the hidden layer after processing. The stimulus produces the final result. If there is a correct result, the correct result is compared with the result, and the error is obtained. Then the feedback weights of the link weights in the neural network are feedback-corrected, thereby completing the learning process [4]. This is the feedback mechanism of the BP neural network, and it is also the source of the name of the Back Propagation: the use of backward feedback learning mechanisms to correct the weights in the neural network, and ultimately achieve the purpose of outputting correct results!

\subsection{Feed Forward Neural Network Algorithm}

Feed forward neural network has a simple structure and is widely used. It can approximate arbitrary continuous functions and square integrable functions with arbitrary precision. Moreover, any finite training sample set can be precisely implemented. From a system point of view, the feedforward network is a static nonlinear mapping. Complex nonlinear processing capabilities can be obtained through complex mapping of simple nonlinear processing units. From a calculation point of view. Lack of rich dynamic behavior. Most feed forward networks are learning networks, and their classification capabilities and pattern recognition capabilities are generally stronger than feedback networks. Feedforward neural networks are divided into single-layer neural networks and multi-layer feed-forward neural networks.

A single-layer feedforward neural network is the simplest type of artificial neural network. It contains only one output layer. The value of the node on the output layer (output value) is directly obtained by multiplying the input value by the weight value. Remove one of the elements for discussion, and the input-to-output transformation relationship is:

$$
\begin{aligned}
& s_{j}=\sum_{i=1}^{n} w_{j i} x_{i}-\theta_{j} \\
& y_{j}=f\left(s_{j}\right)=\left\{\begin{array}{l}
1, s_{j} \geq 0 \\
0, s_{j}<0
\end{array}\right.
\end{aligned}
$$

In the above formula, $x=\left[x_{1}, x_{2}, \ldots, x_{n}\right]^{T}$ is the input feature vector, which is the classification result according to different features.

A multilayer feedforward neural network has one input layer, one or more hidden layers in the middle, and one output layer. The relationship between input and output transformations in a multilayer perceptron network is:

$$
\begin{gathered}
s_{i}^{(q)}=\sum_{j=0}^{n_{q-1}} w_{i j}^{(q)} x_{j}^{(q-1)},\left(x_{0}^{(q-1)}=\theta_{i}^{(q)}, w_{i 0}^{(q-1)}=-1\right) \\
x_{i}^{(q)}=f\left(s_{i}^{(q)}\right)=\left\{\begin{array}{l}
1, s_{i}^{(q)} \geq 0 \\
-1, s_{i}^{(q)}<0
\end{array}\right. \\
i=1,2, \ldots, n_{q} ; j=1,2, \ldots, n_{q-1} ; q=1,2, \ldots, Q
\end{gathered}
$$

Each layer at this time is equivalent to a single-layer feed-forward neural network, such as to the first layer, which forms a hyperplane of dimensions. It linearly classifies the input patterns of this layer, but due to the combination of multiple layers, a more complex classification of input patterns can be achieved in the end [5]. 


\subsection{Neural Network Control During Car Shifting}

In order to make automotive automatic transmission (AMT) shift change to adapt to road conditions and vehicle condition, smooth shift to better meet the automotive and fuel economy requirements, the neural network is proposed three cars AMT shift control parameters.

Taking the ZL50 wheel loader as a prototype, the load factor $\beta$ is defined to describe the amount of engine torque and power consumed by the working oil pump, and it becomes a variable in the continuous dynamic variable speed mathematical model of the X6130 diesel engine. [Alpha] to the accelerator opening degree, load factor beta], $v$ is the speed control parameter, to obtain three new parameters Shift Schedule The traction characteristics; Novel Three curves obtained parameter SHIFT SCHEDULE The energy efficiency of the torque converter, and at different The gear speed points of each gear obtained by the load factor are fitted, and the new three-parameter dynamic shift law and the energy-saving shift law are calculated for different accelerator opening degrees and different load factors. Under the environment of MATLAB/Simulink, the simulation model of the vehicle hydraulic mechanical transmission system and the control model of the automatic transmission system were established. Through simulation experiments, the three-parameter shift control of the neural network of the AMT automobile was compared with the shift schedule and the energy-saving shift schedule and the two Parameters (accelerator opening, speed) shift dynamics and economy.

The tests show that the use of neural network three parameter shifting is more consistent with the driver's shift experience and habits than the use of neural network two-parameter shifting, and the gear switching surface changes smoothly, which is more simple and easy to implement than the traditional calculation method to solve the shift schedule.

\section{Test Results and Discussion}

\subsection{Clutch Implementation Structure}

The clutch mechanism is quickly separated and pressed in a fast-slow-fast speed control tracking test. The results are shown in Figure 3 and Figure 4. The clutch rapid separation time is $0.4 \mathrm{~s}$, which can track the target displacement well, and the mechanism is sensitive and can fully meet the requirements. Clutch control needs.

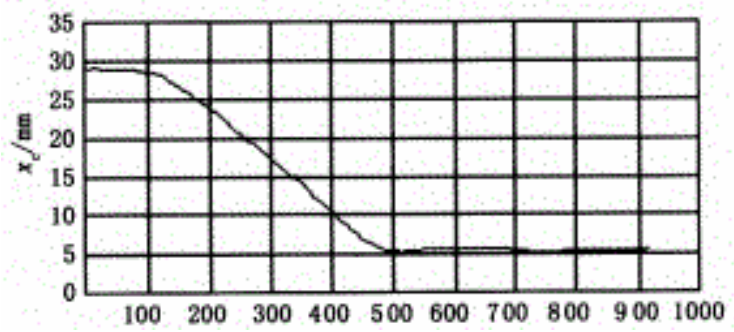

Figure 3. Clutch quick separation curve

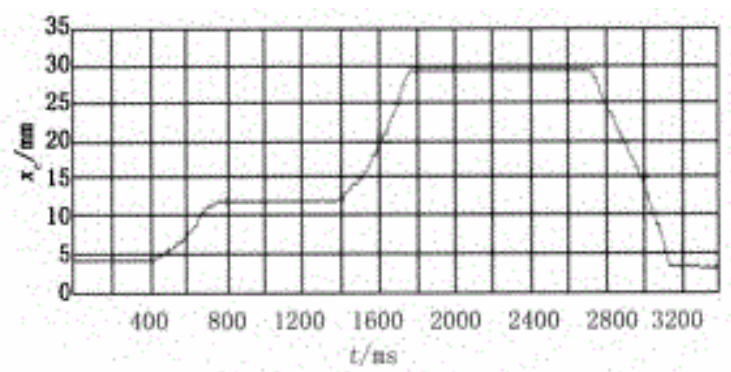

Figure 4. Clutch engagement curve

\subsection{Select Shifting Mechanism}

Through the static continuous shift test of the shifting mechanism, it can be concluded that: 1st gear to 2 nd gear and 3rd gear to 4th gear do not require gear selection process, the total gear shift time is $0.3 \mathrm{~s}$, and 2 nd gear to $3 \mathrm{rd}$ gear and 4 th gear The gear selection process is required from gear 5 
to gear 5 . The total gear shift time is increased by $0.1 \mathrm{~s}$. The response of the motor to the control signal is very fast and it can fully meet the system response speed requirements [6].

\section{Conclusion}

In this paper, the BP neural network is applied to the AMT car control system, and the off-line training is performed based on a large number of empirical values. Finally, the result that the car gear shifting and the clutch combining effect meet the actual demand is achieved.

\section{References}

[1]. Zhai Qun. Study on the Method of Automatically Finding and Selecting Shift Spaces in Electric AMT [J]; Journal of Guangxi University for Nationalities (Natural Sciences Edition); 2012-03.

[2]. Zhang He. Shi Xiaohui; Zou Xihong. Design of multi-axis road simulation test bench for AMT actuator [J]; Mechanical Transmission; 2012-09.

[3]. Yuan Xin. Research on Design Method of AMT Automatic Transmission for Passenger Cars [J]; Mechanical Management and Development; 2012-01.

[4]. Cheng Jin; Zhu Xiaomin; Xu Jing. Sun Mingjun; Duan Mingbiao; Zhang Xi. Design of Test System for AMT Speed Selector Pump Motor Unit Test Bench [J]; Manufacturing Automation; 2011-21.

[5]. Yin Xiao feng. Study on temperature adaptive neural control of working process of AMT clutch [D]. Jilin University of Technology, 2014.

[6]. Bao Jinfeng. Study on New Three-Parameter Shifting Rules of Construction Vehicles [D]; Jilin University; 2011. 